July 30, 1990

LBL-28574

EXPANDED

\title{
A QUANTUM THEORY OF THE MIND-BRAIN INTERFACE •
}

\author{
Henry P. Stapp \\ Theoretical Physics Group \\ Physics Division \\ Lawrence Berkeley Laboratory \\ 1 Cyclotron Road \\ Berkeley, California 94720
}

LBL- -28574

DE91 004431

\begin{abstract}
The Heisenberg quantum mechanical conception of nature is extended and applied to the brain. Strict adherence to the principle of parsimony, and to quantum thinking, produces naturally, on the basis of an overview of brain operation compatible with the information provided by the brain sciences, a unified description of the physical and mental aspects of nature that can account in principle for the full content of felt human experience.
\end{abstract}

Invited Presentation to the Conference: Consciousness within Science, Cole Hall, University of California at San Francisco, Feb. 17-18, 1990.

- This work was supported by the Director, Office of Energy Research, Office of High Energy and Nuclear Physics, Division of High Energy Physics of the U.S. Department of Energy under Contract DE-AC03-76SF00098.

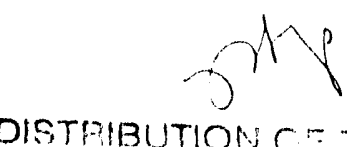




\section{Contents}

1. The Origin of the Problem: Classical Mechanics.

2. A Quantum Ontology

3. The Functioning of the Brain: An Overview

4. Incorporation of Quantum Mechanics

5. Brain and Consciousness

6. Qualia: The Experiential or Felt Quality of Actual Events

7. Comparison to Other Treatments

8. Related Philosophical Issues

9. Summary 


\section{The Origin of the Problem: Classical Mechanics}

Advances in science often unify conceptually things previously thought to be unconnected. Thus Newtonian mechanics unified our understanding of stellar and terrestial motions, and Maxwell's theory unifed our understanding of e'ectro-magnetic phenomena and light. Einstein's special theory of relativity unified our concepts of space and time, and his general theory unified our conceptions of spacetime and gravity. My thesis here is that the integration of consciousness into science requires considering together two outstanding fundamental problems in contemporary science, namely the problem of the connection between mind and brain, and the problem of measurement in quantum theory. Each of these problems concerns the interface between two domains of phenomena that are currently described by using different conceptual systems: mind and brain are described in psychological and physical terms, respectivel; ; whereas the measurement problem in quantum theory is to reconcile the concepts of classical physics that are used to describe the world of visible objects with the concepts of quantum theory that are used to describe the world of atomic processes. In each case the problem of constructing a coherent overarching conceptualization appears to be so intractable that many scientists have judged the problem to be a pseudo problem not suited to scientific study. However, technological advances are now providing data that bear increasingly on the interfaces between the domains that had heretofore been empirically separate. Given these new data, and the prospect of more to come, science can now profitably take up the challenge of providing a conceptual framework that unifies the mental, physical, classical, and quantal aspects of nature.

William James highlighted the seemingly intractable character of the mindbrain problem with the following two quotations:!)

Suppose it to have become quite clear that a shock in consciousness and a molecular motion are the subjective and objective faces of the same thing; we continue utterly incapable of uniting the two so as to conceive the reality of which they are the two faces. (Spencer) and

The passage from the physics of the brain to the corresponding facts of consciousness is unthinkable. Granted that a definite thought 
and a definite molecular action in the brain occur simultaneously; we do not possess the intellectual organ, nor apparently any rudiment of the organ, which would allow us to pass, by a process of reasoning, from one to the other. (Tyndall)

In commenting on this issue James clearly recognized that the problem was with the concepts of classical physics. Referring to the scientists who would one day illuminate the problem he said:

the necessities of the case will malie them "metaphysical". Meanwhile the best way in which we can facilitate their advent is to understand how great is the darkness in which we grope, and never forget that the natural-science assumptions with which we started are provisional and revisable thirigs. ${ }^{2)}$

James evidently foresaw, on the basis of considerations of the mind-brain problem, the eventual dislodgement of classical mechanics from the position it held during his day. We now know that classical mechanics fails at the atomic level: it has been superseded by quantum mechanics.

That classical mechanics is not capable of integrating consciousness into science is manifest. Classical physics is an expression of Descartes' idea that nature is divided into two logically unrelated and noninteracting parts, mind and matter. However, the integration of consciousness into science requires, instead, a logical framework in which these two aspects of nature are linked in ways that can account for both the observed influence of brain processes on mental processes, and the apparent influence of mental processes on brain processes.

Brain process depend in a sensitive way upon atomic processes. Hence a quantum mechanical treatment is mandated in prizciple. However, the brain has a bier archical structure, with larger structures being built from smaller ones, and as one moves to higher levels the concepts of classical physics seern to work increasingly well. Since consciousness appears to be a high-level process one might think that it should be comprehended within the conceptual framework of classical physics. In support of this idea some scientists have noted that, even in nonbiological systems, as one moves to higher levels of organization new 
structures often emerge that exercise effective control over lower-level processes. Thus it is argued that just as a "vortex" can, within the conceptual framework of classical physics, emerge as an entity that controls the motions of the molecules from which it is built, so might there emerge, from a stratum of brain activities completely compatible with the concepts of classical physics, a "consciousness" that controls lower-level brain processes.

There is, however, an essential conceptual difference between consciousness and a system such as a vortex that is compatible with the concepts of classical physics. The essential characteristic of consciousness is that it is felt: it is felt experience; felt awareness. Any system that is compatible with the concepts of classical physics can be described, insofar as its physical behavior is concerned, as composed of the physical elements provided by classical physics, such as atoms, and electro-magnetic fields. However, the description in terms of these elements does not, by itself, specify whether the system has an appended experiential aspect - a feel. Nature may elect to add feel, but the classical physicists can consider the purely physical version without any added quality of feel, and this latter version behaves, according to the precepts of classical physics, in exactly the same way as the one with feel. Thus within the framework of classical physics feel is, per se, nonefficacious: it has no effect on the physical world.

This problem has been clearly understood for hundreds of years, and is the core of the mind-brain problem.

It is only recently that the brain sciences have amassed enough data to make feasible a serious effort to understand the dynamics of the mind-brain connection within the framework of the basic laws of physics. An adequate classical-physics treatment of the mind-brain problem is not possible, for the reason discussed above. On the other hand, the application of quantum mechanics appears to be blocked by three major technical problems.

The first problem, which has already been mentioned, is that quantum theory is primarily a theory of atomic processes, whereas consciousness appears to be connected with macroscopic brain activities, and macroscopic processes are well described by classical physics.

The second problem is that, due to a failure of an essential condition of isolation, quantum theory, as developed for the study of atomic processes, does 
not apply to biological systems, such as brains.

The third problem is that the orthodox Copenhagen interpretation of quantum theory instructs us to regard the quantum formalism as merely a set of rules for calculating expectations about our observations, not as a description, or picture, of physical reality itself. However, without a description of physical reality consciousness becomes a puzzle within an enigma.

Any acceptable quantum mechanical treatment of the connection between mind and brain must resolve these three major technical problems. In the treat,ment to be described here the resolution of the third problem resolves automatically also the other two.

\section{A Quantum Ontology}

The mathematical concepts in quantum theory are fundamentally different from those of classical physics. This difference makes it difficult to form a unified conception of nature. The Copenhagen strategy for circumventing these conceptual difficulties, by settling for a set of computational rules connecting human observations, rather than striving to comprehend the nature of the underlying reality, was strongly opposed by Einstein, Schroedinger, and many other principal contributors to the development of quantum theory. However, those critics were unable to put forth any alternative proposals. Eventually Werner Heisenberg, one of the chief architects and strongest defenders of the Copenhagen interpretation, did try to form a coherent pictures of what is actually happening.

In Heisenberg's picture, which is the one informally adopted by most practicing quantum physicists, the classical world of material particles, evolving in accordance with local deterministic mathematical laws, is replaced by the Heisenberg state of the universe. This state can be pictured as a complicated wave, which, like its classical counterpart, evolves in accordance with local deterministic laws of motion. However, this Heisenberg state represents not the actual physical universe itself, in the normal sense, but merely a set of "objective tendencies", or "propensities", connected to an impending actual event. The connection is this: for each of the alternative possible forms that this impending event might take the Heisenberg state specifies a propensity, or tendency, for the event to take that form. The choice between these alternative possible forms is 
asserted to be governed by "pure chance", weighted by these pr spensities.

The actual event itself is simply an abrupt change in the Heisenberg state: it is sometimes called "the collapse of the wave function". The new state describes the tendencies associated with the next actual event. This leads to an alternating succession of states and events, in which the state at each stage describes the propensities associated with the event that follows it. In this way the universe becomes controlled in part by strictly deterministic mathematical laws, and in part by mathematically deñned "pure chance".

The actual events become, in Heisenberg's ontology, the fundamental entities from which the evolving universe is built. The properties of these actual events are determined by the quantum formalism. These properties are remarkable: they lead to a quantum world profoundly different from the one pictured in classical physics.

Each Heisenberg actual event has both local and global aspects. Locally, each such event acts over a macroscopic domain in an integrative fashion: it actualizes, as a unit, some integrated high-level action or activity, such as the fring of a Geiger counter. This essential quality of the actual event to grasp as $a$ unit, and actualize as a whole, an entire high-level pattern of activity injects into the quantum universe an integrative aspect wholly lacking in the classical conception of nature. This fundamentally integrative action of the Heisenberg actual event is the foundation of the quantum theory of consciousness developed here.

Each actual event has also a global or universal aspect: its action is not wholly confined to any local region, but extends to distant parts of the universe. These two intertwined aspects irise from the fact that the Heisenberg actual event is represented within the quantun formalism by the change induced in the Heisenberg state of the universe by the action upon it of a localized operator. This change in the state of the univere, although induced by the action of a localized operator, produces a global change in the tendencies for the next actual event. Thus each actual event is a global change in the tendencies for the next actual event.

By introducing in this way a quantum ontology, and thus departing from the purely epistemological stance of the strictly orthodox Copenhagen inter- 
pretation, one can remove the subjective human observer from the quaritum description of the physical world and speak directly about the actual dispositions of the measuring devices, rather than the knowledge of the observer. Thus the moon can be said to be "really there" even when nobody is looking. And Schroedinger's cat is, actually, either dead or alive. More importantly, the degrees of freedom of a biological system that correspond to its macroscopic features can be considered to be highly constrained, and to specify a classical framework, or matrix, within which one can consider the atomic processes that are essential to its functioning.

This useful ontology has two defects. The first is its run-away ontology: the supposedly actual things to which the tendencies refer consist only of shifts in tendencies for future actual things, which consist, in turn, only of shifts in tendencies for still more distantly future things, and so on ad infinitum: each actuality is defined only in terms of possible future ones, in a sequence that never ends.

The second defect is the omission from the description of nature of the one thing really known to exist: buman thought.

These two difficulties fit hand-in-glove: the first is that some authentic actual things are needed to break the infinite regress; the second is that some authentic actual things have been left out.

These considerations motivate the first basic proposal of this work, which is to attach to each Heisenberg actual event an experiential aspect. The latter is called the feel of this event, and it can be considered to be the aspect of the actual event that gives it its status as an intrinsic actuality.

The central question then becomes: What principle determines the structure of the feel of an actual event? More narrowly: How is the structure of human experience connected to the structure of human brain processes?

The answer, according to the present theory, is this: Each human experience has a compositional structure that is isomorphic to the compositional structure of the actual brain event of which it is the feel.

To understand the nature of these two compositional structures one must look closely at brain processes and psychological processes. We begin by giving a general overview of the former. 


\section{The Functioning of the Brain: An Overview}

The primary function of the brain is to gather information about both its environment and the body, to formulate possible plans of action, to choose a single plan of action, and to oversee the execution of that plan. Various patterns of neural excitation become activated in the course of these activities. These patterns must presumably represent, among other things, the information that needs to be processed, such as the sensed state of the body and the environment, and the programs for coordinated motor action.

Gerald Edelman ${ }^{4)}$ has given recently a scientifically based account of how the brain could have: (1), evolved under natural selection; (2), developed during its individual growth; and (3), become conditioned by its individual history, in such a way as to allow these features that need to be processed to become represented by patterns of neural excitations. One key ingredient is the idea of the facilitation of such patterns by physical changes at the synaptic junctions. This process permits certain recurring patterns of excitations in the cerebral cortex that are originally weakly activated by a particular neural activity to become strongly and selectively activated by that activity. Facilitation also permits association, whereby the excitation of parts of a facilitated pattern activates, under certain conditions, the rest of that pattern. This association process provides a neural mechanism for retrieval of memories.

To do its job the brain must evidently possess a representation of the body and its environment. I call this representation the body-world schema. A lizard, or a frog, as it watches a moving insect, is, by its attention, continually updating parts of its body-world schema. Quite generally, a basic element of brain operation is the periodic updating, by attention to particular details, of parts of the body-world schema.

When I choose to raise my arm I do not consciously instruct each muscle. I mentally raise my arm to its intended place, and unconscious processes execute the implied instruction. Thus we evidently possess a "projected body-world schema" whose content is akin to that of the "current body-world schema", but which specifies a goal or intention, rather than the current state of affairs. It shares with the current body-world schema the feature that its contents are periodically updated in response to conscious acts of attention. 
Each item in the body-world schema (current, projected, and historical) has a certain key part, which is part of the directive, or instruction, that led to the placement of that item in the schema. Thus if I consult my body-world schema to find out what I just saw on my right, the instruction by which I can reconfirm or update that item is immediately available through association: upon releasing an inhibition this instruction becomes carried out by the unconscious levels of processing.

When I choose to raise my arm I also generally choose, or intend, at the same time, to monitor its motion. Thus, just as for the current body-world schema, an item placed in the projected body-world schema can generally contain an instruction of the same kind as the instruction that produced that item. This instruction placed in the projected body-world schema will, if not amended, normally be carried out at the appropriate time by the unconscious processes.

It appears from these considerations that the brain can, under suitable conditions of alertness, sustain a "top-level process" with the following three general characteristics:

1. Its elements are events that actualize instructions to lower-level processes.

2. These instructions cause the lower-level processes to gather information, prepare for and execute actions, and construct the next top-level instruction.

3. Each top-level instruction is an updating of the body-world schema, or of some generalization of that schema.

At the neural level this sort of arrangement can be implemented by a category of patterns of neural excitations thai I call "symbols". Each top-level instruction consists of a collection, or "chord", of these symbols. Each such symbol when "released" (e.g., by blocking some inbibitory signals) tends to activate, by association, the lower-level processes that it symbolizes.

This general picture of brain operation, which will be amplified later, appears compatible with the growing body of evidence coming from the brain sciences (See ref. 27). I shall not review the evidence here, but will simply accept this overall picture and proceed to explore the impact of treating quantum mechanically certain important atomic processes that occur in the alert brain. 


\section{Incorporation of Quantum Mechanics}

An element of brain dynamics where atomic processes play a key role is the release of a vesicle containing neurotransmitter into a synaptic junction. Our theoretical picture ${ }^{5)}$ of this process is that an action-potential pulse opens channels for calcium ions, which then migrate by diffusion to release sites. Several such ions must attach at a site to effect the release of the vesicle.

In the model of ref. 5 a calcium ion travels about $50 \mathrm{~nm}$ in a time of about $200 \mu s$, on its way from channel exit to release site. Simple estimates of the uncertainty principle limitations upon body-tempera- ture calcium ions diffusing in this way show that the wave packet of the calcium ion must grow to a size many orders larger than that of the calcium ion itself. Hence that the idea of a single classical trajectory becomes inappropriate: quantum concepts must in principle be used.

According to quantum theory the quantum state generated by this process of diffusion is a complex multiparticle state whose one-particle probabilities should approximate the probabilities given by the classical calculation.

The probability for an action--potential pulse to release a vesicle at a cortical synapse appears to be about $50 \% .^{6}$ ) If, in some small time window (say a fraction of a rillisecond), $N$ synapses receive action-potential pulses then there will be $2^{N}$ alternative possible configurations of vesicle releases, each with a roughly equal probability. Each alternative possibility is represented in the evolving quantum mechanical wave function.

The brain is a highly nonlinear system with feedback. Classical computer simulations ${ }^{7}$ show that the macroscopic state into which it will evolve is very sensitive to small variations at the synaptic level. It is therefore, I think, virtually inconceivable that a variation over the $2^{N}$ alternative possible configurations of vesicle releases could, in general, have no influence on the eventual macroscopic state into which the system evolves. Nonlinear systems are generally very sensitive to smal' changes, and there is no reason to believe that the brain could be totally insensitive to such differences. Thus a universe containing a conscious brain, represented quantum mechanically, must be expected to evolve into a state that represents a superposition of macroscopically different alternative possibilities for the brain, provided there is no actual event that reduces 
the state to one that is not a superposition of this kind.

This raises the key question: At what point does an actual event intervene?

This issue was addressed by John von Neumann in an analysis ${ }^{8}$ that constitutes the foundation of the quantun theory of measurement. von Neumann considered a sequence of measuring devices, with the first one measuring the atomic system, and each other device measuring the response of the one before it, with the last one being, conceptually at least, some innermost level of the brain. He showed that, for his idealized case, it made hardly any difference at all at which point the actual event intervened to select one of the several macroscopically different possibilities: the quantum mechanical probabilities were virtually independent of where the "Heisenberg cut" was drawn between the "quantum system" and the classically described device that was measuring it.

I shall exploit von Neumann's result by assuming that in the alert brain the main actual events occur at the point where a choice is made between alternative possible instructions in the top-level process. Since tcn-level instructions generally initiate large and differing responses by the lower-level processing mechanisms, this assumption is analogous to Heisenberg's assumption for inanimate objects that the actual event occurs only at a high level, where it chooses between states corresponding to macroscopically different actions of the object, such as the firing or nonfiring of a Geiger counter. Human conscious events are assumed to be the feels of these top-level events, which actualize macroscopic patterns of neural activity. We now have in place a general description of brain operation compatible with quantum theory, and can pose the question of the connection of brain to consciousness.

\section{Brain and Consciousness}

We are not conscious of what is going on in our brains. We are conscious of, for example, Beethoven symphonies, and sunsets. How can such a felt experience be the "feel" of some evenis in the brain?

To start with something simpler than a Beethoven symphony consider a triangle: Why, when we look at a triangle, do we experience three lines joined at three points, and not some pattern of neuron firings?

To answer this question let us consider first Edelman's explanation of how the visual cortex comes to be organized. The problem is this: the growth of the 
neurons connecting the retina to the visual cortex is not completely determined by genetic programming: there is a great deal of contingency. But then how does the structural information present at the retina get properly reconsitute at the cortex, rather than becoming hopelessly scrambled by the randumness of the neural connections?

The answer is that the saccadic movements of the eye cause the neurons that receive signals from adjacent retinal regions to receive temporally correlated signals. The resulting spatially distributed but temporally correlated patterus of excitation in the visual cortex then become automatically associated, by the facilitation process. Thus some of the structure at the retinal level becomes mapped into a spatially distribution analog structure in the realm of the cortical patterns of exitaition.

Building up from this initial organization, initiated by the saccauic eye movements, repetitious patterns occuring at the retina facilitate corresponding patterns in the cortex.

Thus even though the neural wiring is somewhat haphazard, the process of facilitation nevertheless automatically establishes analogs of attended or recurring retinal patterns within the realm of the cortical natterns of excitations.

Patterns present in the visual cortex become associated, in the same way', with the neural accompaniments of those motor actions that bring them into being. Thus recurring features of the external visual scene will come ' 0 be associated with complex patterns of excitations that include the patterns that produce the motor actions that allow these features to be sensed.

Due to this mapping of structure the cortical patterns generated by attention to the exte:nal triangle will be "congruent" to the external triangle. For example, the adjacency properties of the points along the three lines of the triangle will have their symbolic representations among the cortical patterns originally facilitated by the saccadic eye movements. Similarly, the various other perceived structural features of the external triangle will be represented by symbols that have been previously constructed by brain processes to represent such connections.

The act of attending to the external triangle implants this symbolic representation of the externa' triangle into the body-world schema. More specif- 
cally, this act of attending leads to an actual event that updates the body-world schema by actualizing an integrated chord of symbols that is "congruent" to the external triangle, in the sense that it will contain symbols that are the analogs of the various structural features that characterize the external triangle itself.

It might seem that this shift from the external triangle to a congruent inner representation has not helped at all, but only made things worse. Even if we grant the congruency property the question remains: Why do we experience the triangle rather than the firings of neurons? We do not wish to introduce an homunculus that surveys the brain, and is able to decipher its complex activity and see a triangle.

This deciphering problem arises, however, only if one slides back to the classical concepts. In the quantum ontology a brain attending to an external triangle is not performing the retrograde act of transforming an actual external triangle into some congruent structure of particle motions, which must then be deciphered to be perceived as a triangle. Rather it is transforming the external triangle, which exists only as a pattern of disjoint events and tendencies, into a single event that actualizes, in integrated form, an image of the structural connections that inhere in the perceived triangle. The brain, therefore, does not convert an actual whole triangle into some jumbled set of particle motions; rather it converts a concatenation of separate external events into the actualization of some single integrated pattern of neural activity that is congruent to the perceived whole triangle. The central question is then: Why is the actualizing of this integrated pattern of activity felt as the perceiving of the triangle? More generally: Why do brain events feel the way they do?

\section{Qualia: The Experiential or Felt Quality of Actual Events.}

The present theory asserts that each human conscious experience is the feel of an event in the top-level process occurring in a human brain. This brain process is asserted to consist of a sequence of Heisenberg actual events called the top-level events. Each such event actualizes some macroscopic quasi-stable pattern of neural activity. The pattern actualized by a top-level event is called a symbol. It normally consists of set of other symbols, called its components, linked together by a superposed neural activity.

Actualizing a symbol $S$ engenders enduring physical changes in the synapses 
(facilitation) that cause any subsequent actualization of any coinponent of $S$ to create a pattern of dispositions for the activations of the other components of $S$ (association). Thus the actualization of any symbol $S$ creates a pattern of dispositions for the activation of all symbols having a component that is a component also of $S$.

The actualization of any symbol $S$ thus produces tendencies for the activation of various collections of symbols. One such collection, $C$, may be far more strongly disposed to activation than the others. Then the actualization of $S$ constitutes an instruction for the artualization of that collection of symbols $C$.

Due to quantum indeterminary many alternative possible collections $C$ must have nonzero weight. The next top-level event actualizes one collection, together with a superposed structure of neural activity that grows up around it and gives the whole pattern stability and distinctiveness, allowing it to stand out from the chaotic continuum of background activity and be actualized as a distinct quasi-stable pattern of neural activity. The full set of symbols, and of dispositions of symbols to activate symbols, created during the life of the brain by the top-level process, is called the generalized body-world schema. The bodyworld schema mentioned earlier is an integral part of it. Each top-level event augments the generalized body-world schema, and is therefore an updating of it.

The generalized body-world schema is an organizational structure in which all symbols are effectively stored, in latent form, for later retrieval by crossreferencing. The retrieval mechanism is presumably this: if a symbol $S$ has a disposition to be activated by several symbols, then the simultaneous actualization of these several symbols will cause $S$ to be activated more quickly, and hence become actualized before the symbols less strongly disposed to activation reach the threshold for possible actualization.

This retrieval mechanism can allow brain process to actualize, by crossreferencing, the symbol that represents, for example, the occupant of a certain place at a certain time, without interference from the symbols representing the occupants of that place at other times, or the occupants of other places at that time; and to actualize the symbol that represents the place where an object represented by a certain symbol is located at a certain time, without interference from the symbols representing the locations of that object at other times. The 
generalized body-world schema thus becomes the physical basis for the longterm memory system. The top-level proceis is the generator of this memory system.

We may now state an essential point: Each top-level event actualizes a symbol, and this symbol has components that are themselves symbols. Thus each top-level event is represented by a symbol that has a compositional structure: it has components that are entities of the same kind as itself.

Consider next the mental side. The structure of mental states has been extensively studied. I accept the conclusions of William James, who cites with strong approbation the following quotation: "Our mental states always have an essential unity, such that each state of apprehension, however variously compounded, is a single whole of which every component is, therefore, strictly apprehended (so far as it is apprehended) as a part. Such is the elementary bases from which all our intellectual operations commence" ${ }^{n \text { ) }}$

A component of a thought, so far as it is apprehended, is itself a possible thought. Thus each thought has a compositional structure: it has components that are entities of the same kind as itself. Our basic principle is that the compositional structure of the feel of a top-level event is isomorphic to the compositional structure of the symbol actualized by that event: there is a oneto-one mapping of symbols to feels, and this mapping preserves compositional structure.

The fundamentally integrative character of the Heisenberg actual event enters here in a critical way. The Heisenberg event grasps as a whole an entire integrated pattern of physical activity. This essential unity of the actualized physical state accords with the essential unity of its mental counterpart.

William James has described the profound conceptual inadequacy of classical mechanics - as a basis for understanding the connection between brain and mind - that is so satisfactorily resolved at this point by quantum theory. Having emphasized the essential unity of each thought, and a first difficulty that arises from it, James goes on to say: "The second difficulty is deeper still. The 'entire brain-process' is not a physical fact at all. It is the appearance to an onlooking mind of a multitude of physical facts. 'Entire brain' is nothing but our name for the way in which a million of molecules arranged in certain positions may affect 
our senses. On the principles of the corpuscular or mechanical philosophy, the only realities are the separate molecules, or at most the cells. Their aggregation into a 'brain' is a fiction of popular speech. Such a fiction cannot serve as the objectively real counterpart to any psychic state whatever. Only a genuine physical fact can so serve. But the molecular fact is the only genuine physical fact $-{ }^{n 10)}$

In the quantum ontology the only genuine physical facts are the actual events. Hence some actual event must 'serve as the objectively real counterpart to [each] psychic state.' But in this case the essential unity of the physic state - so incomprehensible within reductionist classical thought - mirrors the essential unity of its physical counterpart. In both case the ontological progression is from the ontologically fundamental wholes to their ontologically subordinate components, rather than from presumed ontologically fundamental elements to assemblies thereof. This shift from synthetic ontology to analytic ontology is the foundation of the present work.

A fundamental feature of experience is the feel of the 'flow of consciousness', or the 'perception of time'. On the other hand, each actual event is ontologically distinct from all others, and its feel is the feel of itself alone. Thus the "present" mental event is the feel exclusively of the "present" physical event; it has no access to past physical events.

But how, then, does one account for the 'flow of consciousness' and the 'perception of time'. These phrases refer to an extensively analyzed empirical structure described in rough terms by William James in the following way: "If the present thought is of $A B C D E F G$, the next one will be of $B C D E F G H$, and the one after that of CDEFGHI - the lingerings of the past dropping successively away, and the incomings of the future making up the loss". ${ }^{11}$

According to this picture, each immediately present mental event contains within itself a sequence of parts perceived as "temporally" ordered.

This "temporal" structure of each mental event evidently arises, in part, in the following way: due to the quasi-stable character of symbols the symbol actualized by a top-level event will generally have among its components, many of the components of the symbol actualized by the preceding top-level event: the set of components of the new symbol will include many of the components 
of its predecessor, together with some new symbols. Thus the feel of the new event will have components that correspond to components of earlier events.

If someone recites quickly an unfamiliar sequence of four numbers an attentive listener can readily repeat the sequence, or repeat the part of it starting from any one of its four components. However, reciting the sequence the reverse order requires more effort. Thus there is evidently a dynamical tendency for associations between the temporal slices of a thought to move from any slice to its temporal successor, rather than randomly about. The existence of this tendency means that the superposed structure of the symbol, which creates the dispositional "associations" between its components, must give larger dispositions to the associations that run forward in the "temporal" ordering. Since this enveloping neural structure tends to recreate the earlier temporally ordered patterns of activity, such a biasing for "forward" association is to be expected. It will be accommodated in our representation of the compositional structure of a symbol in terms of its components by allowing both a " + " composition that is commutative $[a+b=b+a]$ and also a "sequential product" that is noncommutative and nonassociative $[(a b c d) \neq(a b d c),((a b c)(d e f)) \neq(a b c d e f)]$. The " + " composition combines symbols without "temporal" biasing, and the sequential composition combines symbols with "temporal" biasing. Thus the brain event that follows upon the hearing of the spoken sequence $(5,6,2,8)$ is represented by (5628), and it is felt as the heard sequence $(5,6,2,8)$. Here I have used the same numeric symbols to represent the spoken words, the components of the symbol actualized by the top-level brain event, and the components of the feel of that event.

James' picture of a marching sequence of fixed letters is only a first approximation. Each actualized symbol creates dispositions for the activation of various symbols that were actualized together with itself in earlier top-level events. Thus as one of James' letters marches through the sequence of successive events its original symbolic counterpart becomes embellished by an expanding network of symbols, consisting of symbols that were actualized together with it during earlier top-level events. The feel tied to the marching letter consequently becomes embellished by the feels of these earlier events: its "meaning" becomes enlarged and sharpened by the agglutination of feels associated with related past events.

The symbols are quasi-stable structures with fatigue characteristies that 
cause them eventually to fade out. Thus after an initial period of intensity, accompanied by a growing sense of "meaning", the feel tied to any fixed letter in James' picture will begin to fade out, and it will eventually die away. James has described this waxing and waning of the intensity and sharpness of the "temporal" components of a present mental state, when it is analysed in terms of the variation of the "temporal" variable: the earlier components are vague, the ones later in the "temporal" sequence are clearer, and the ones still later in this sequence fade away.

The range of possible "meanings", as characterized by the number at possible structural forms of these embellishments, can be huge. James cites evidence that a mental event may have as many as 40 temporally distinguished parts. ${ }^{12}$ ) Suppose there are just ten fundamental symbols, and that all others are formed by simply the sequential compositions of these ten. Then the number of possible embellishments generated in the first 20 steps, e.g., before the fading sets in, is $10^{20}$.

Embellisbment leads to "meaning" because the embellished symbol is experienced as a felt structure of feelings each of which corresponds to a related past event: an observed "bicycle" comes to be associated with a structure of feels in which are imbedded childhood experiences of locomotion, spills, adventures, etc.,: i.e., of what a bicycle led to in the past, and hence might lead to again.

These "meanings" arise, however, only from the structural content of the symbol: the ten basic symbols act as undefined symbols from which all the structures are built, but the 10 ! permutations of these ten basic symbols leave the internal structural content unchanged: all connections between feels are left unchanged by these permutations. Thus the possible shades of meaning number, in principle, $10^{20} / 10$ !, in this example.

The distinction being emphasized here is between the elemental, or absolute, units of experience, such as the immediate direct experience of redness, or of the pitch of high $C$, and the meanings of symbols that arise from their compositional structures. The former are the feels of certain actualized patterns of neural activity, and would be different if the patterns of neural activity representing these symbols were different. The former reside in the internal structural composition of the symbol and would be left unchanged if the feel of all symbols were shifted in a way that maintained the feel of "nearness" that feels can have 
to one another.

The dynamical process of embellishment considered above, in which the symbolic counterpart of each "letter" in the temporal sequence develops associations by itself, as if it developed in isolation from the other symbols actualized together with it, is an over simplification: symbols actualized together act together; they act as combined dispositions for the activation of other symbols. It is this capacity of the different temporal components of a single top-level event to act jointly that gives brain process its capacity to compare and combine symbols, and to manipulate them in other ways.

Each normal top-level event contains a background of symbols that persists through the various "temporal" slices into which it is divided. This background of symbols is felt as a persisting background of intentions and other feels, against which the more transitory feels are contrasted. This background constitutes the feel of "self" that pervades each normal human experience.

This felt "self" is simply part of the experience. The only carrier that links these experiences together is the brain: the brain is the only receiver of the experiences. Each experience exists, and has a structure that mirrors the structure actualized in the brain by the event it reifies. What could be more simple and natural?

\section{Comparison to Other Treatments}

Gerald Edelman and John Eccles have recently set forth detailed jroposals concerning the connection between mind and brain. Their proposals, which constitute serious efforts to accommodate, and integrate, the growing body of neurophysiological, neuropsychological, and other relevant scientific data, are compared in this section to the theory described above. Comparison is made also to the positions of Bohr, von Neumann, and Wigner.

\subsection{Comparison to Edelman}

Edelman's theory rests on a comprehensive general account of the development of the brain during evolution, during embryonic grol th, and during the life of the individual person. This account provides a fairly detailed description, based on the relevant scientific data, of the development and functioning of a level of neural processing that is subsumed in my term "lower-level processing". 
According to Edelman's theory these lower-level brain process must contain four specific components if consciousness is to emerge. The first of these components is perceptual categorization, which is a neural process mediated by synaptic change that causes particular patterns of neural activity to become activated by, and hence associated with, particular patterns of signals from sense organs. The second lower-level component deemed necessary for the emergence of consciousness is the functioning of neural pathways dedicated to the incorporation into brain processing of values pertaining to the physiological and other needs of the organism. ${ }^{13)}$ The third necessary lower-level component is memory, which, in this context, is a system property of the brain, mediated by synaptic change, which arises from the continual creation of new patterns of neural activity representing new categories. These new categories, expressed as neural activities: correlate and compare the categories previously created. ${ }^{14)}$ The fourth component of lower-level neural processing deemed necessary for consciousness is a component that effects learning, which is "context-dependent behavioral change governed by positive or negative value under conditions of expectancyn..$^{15)}$

These four components of brain processing can, according to Edelman's theory, function without the occurrence of conscious awareness, i.e., without consciousness. According to Edelman's theory, "Consciousness is the result of an ongoing categorical comparison of the workings of two kinds of nervous organization. This comparison is based on a special kind of memory, and is related to the satisfaction of certain physiologically determined needs as that memory is brought up to date by the perceptual categorizations that emerge from ongoing present experience. Through behavior and particularly through learning, the continual interaction of this kind of memory with present perception results in consciousness. ${ }^{16)}$

The terms "memory" and "perception", as used here, do not in themselves carry any connotation of conscious awareness: they pertain to neural process, as described above. Consciousness is thus claimed to be the result of an interaction between these two components of the unconscious neural processing.

This key process of the emergence of consciousness is described in various places in Edelman's book: " . . . imagine that the various memory repertoire dedicated to the storage of the categorization of past matches of value to percep- 
tual category are [reciprocally] connected to [the neura] syster ij dealing with current sensory input and motor response. By such means, past correlations of category with value are now interactive in real time with current: perceptual categorizations before they are altered by the value-dependent portions of the nervous system. A kind of bootstrapping occurs in which current valuefree perceptual categorization interacts with value-dominated memory before further contributing to alteration of that memory. Primary consciousness thus emerges from a ... recategorical memory (relating previous value-category stquences) as it interacts with current input categories arising from neural systems dedicated to present value-free perceptual categorizations. ${ }^{n 17) ~ " I t ~ i s ~ t h e ~ d i s c r i m-~}$ inative comparison between value-dominated memory involving the conceptual system and the current ongoing perceptual categorization that generates primary consciousness of objects and events" 18)n "the generation of a 'mental image' ... emerges as a result of a series of ... correlations of [perceptual] categories to ... values ..." 19) "The functioning of these key [reciprocal] connections [between past value-category connections and current perceptual categorizations] provides the sufficient condition for the appearance of primary consciousness" 20).

The question arises as to how one is to interpret this claim that this special neural process is a sufficient condition for consciousness to occur. Does this claim mean that the occurrence of consciousness is logically entailed by the occurrence of this neural process?

At the beginning of his book Edelman lists a set of constraints on his undertaking. The first of these is the condition that "Any adequate global theory of brain function must include a scientific theory of consciousness, but to be scientifically acceptable it must avoid the Cartesian dilemma. In other words, it must be uncompromisingly physical and be based on res extensa, and indeed be derivable from them"21)

This condition seems to demand that the emergence of consciousness be derivable from the properties of matter. Edelman accepts "modern physics as an adequate description for our purposes of the nature of material properties" 22) Thus Edelman's demand appears to be that the emergence of consciousness must be actually derivable from physies, or at least from properties of systems describable in principle in terms of the cincepts of physics. This strong interpretation is reinforced by the claim r.ade in the final chapter that "no special addition to 
physics is required for the emergence of consciousness" 23).

If this indeed be the claim then Edelman's account falls short. For the particular neural process that in clairred to be sufficient for the emergence of consciousness is a physical process describable in principle in terms of neural patterns of excitation, and hence, if one ignores the subtleties connected to quantum theory, as Edelman does, in terms of atoms, and electrons, etc. According to the precepts of physics (if quantum effects are ignored) these atoms and electrons, etc., will behave in exactly the same way whether or not a quality of conscious awareness emerges in connection with this particular physical process.

This particular neural process may be connected in some very natural way to some particular quality or kind of awareness. However, that fact, joined to the laws of physics does not entail that this particular quality of awareness must actually come into existence when that physical process occurs. Consequently, the assertion that this quality of awareness does come into existence under those special physical conditions is "a special addition physics": it is not entailed by, or derivable from, the principles of physics.

To the extent that one ignores the effects introduced by quantum theory, and bence adheres to the precepts of classical physics, this extra or added quality of awareness is necessarily nonefficacious: it has no effect on the ongoing neural process. The theory therefore does not succeed in avoiding the Cartesian dilemma, as the initial condition demanded, but introduces a causally disconnected res cogitans.

Edelman has, it appears to me, accepted a tacit assumption that if there is a neural action that functions in a way that is a natural image of the subjective feel of a possible conscious event, then this conscious event will in fact occur if the neural action occurs. This is Edelman's implicit analog of my explicit postulates about feels.

The problem with Edelman's approach is that if one adheres to his demand that the "view of brain function and consciousness should be based on materialist metaphysics"24), and bence rules out quantum physics, and perforce retreats to classical physics, then there is nothing in the physics that singles out these special processes as being in any way special. They are special only because they 
can be associated in a certain way with things outside classical physics, namely possible conscious experiences. But then the claimed connection between these two domains is, from the physics point of view, completely ad hoc. This adhocness is connected to the fact that the conscious awareness, per se, is, within the conceptual framework of classical physics, wholly nonefficacious.

In the Heisenberg quantum ontology, on the other hand, the place where consciousness enters is, from the physics point of view, dynamically singled out, and consciousness is able to become causally efficacious. Consequently, the quantum theory of consciousness comes much closer to filling Edelman's demand that the throry be $t$ ised on res extensa, as described by modern physics, than his theory coes.

\subsection{Comparison to Eccles}

The theory of Eccles ${ }^{25}$ is explicitly dualistic: it postulates a mental entity that interacts with the brain, and that continues to exist after the death and destruction of the brain. This "homunculus" is allowed to influence brain process by exploiting the lack of determinism allowed by quantum theory. Although Eccles' theory thus exploits the freedom introduced by quantum theory, it neither appeals to, nor exploits, the profound conceptual change wrought by quantum theory.

Eccles' theory is fundamentally different fiom the theory proposed here, which explicitly ties every human conscinus event to a corresponding physical event in a human brain. Neuropsychological evidence exists that discriminates, I believe, between Eccles' theory and mine. It comes from the behavior of certain patients who have suffered massive parietal lobe damage, and subsequently exhibit a neglect syndrome: a loss of ability to attend to certain parts of their bodies located contralateral to the damaged area of the brain. Their behaviors suggests that the impairment is more than just a loss of ability to control or sense parts of the body, or even to communicate or speak about them, but is rather is a complete disappearance of any representation of the afflicted part of the body from the patient's repertoire of conscious thoughts: the afflicted part seems simply to disappear from the patient's conception of his body.

Such an effect can be naturally understood as a consequence of elimination of the representation of the afflicted part from the body schema by the destruc- 
tion of the neural basis of the patterns of activity that constitute the symbols that correspond to that part of the body. In the quantum theory of consciousness proposed here the menial universe of each human being consists exclusively of the felt quality of actual events constructed out of the symbols that are the building blocks of the (generalized) body-world schema: consciousness is the felt quality of the manipulating actions of these symbols upon each other. These symbols are thus the currency of consciousness and the destruction of any of them must cause a reduction in the person's mental universe.

A homunculus residing in a separate mental world, and able to survive the death and destruction of the brain, would, presumably, not be itself impaired by the brain damage: its mental universe would be left essentially intact. The damaged brain would be unable to respond as fully to the action of the homunculus upon it, and this impairment would result in problems in communication, and control, and in the reciprocal action of sensing. But the representation of the afflicted part would not disappear from the patient's mental universe itself, as is suggested by the evidence: the patient should not be puzzled to discover that there is a left arm connected to his body; ${ }^{26,27)}$ the patient should "know" that he has a left arm, even though he has recently been deprived by brain damage of the ability to directly sense or control it. Hence he should not be puzzled to discover it.

Some other evidence supportive of the quantum theory, but not necessarily discrimative relative to Eccles' theory, is the data of Libet ${ }^{28)}$ pertaining to the delay in the occurrence of the conscious awareness of a voluntary intention to act, relative to the onset of the neural activity that prepares for the conscious event. The foundations of the quantum theory of consciousness are: (1), the idea that the brain functions to plan, select, and execute single integrated actions; (2), the idea that, due to the unavoidable intrusion of quantum uncertainties into the synaptic processing, and the subsequent amplification of these quantum synaptic processes, the brain functions in a way that is basically similar to a quantum measuring device such as a Geiger counter, in the specific sense that the evolution of the physical system in accordance with the basic local law of evolution (i.e., the Schroedinger or Heisenberg equations of motion) necessarily produces, normally, a state that represents a superposition of macroscopically distinctive states, such as the firing or nonfiring of the Geiger counter, or the activation or nonactivation 
of the neural activities that represent the intention to raise an arm: and (3), the acceptance of Heisenberg position that these two alternative macroscopic possibilities do not both actually occur, in some absolute sense, as is claimed by the competing "many-worlds" interpretation of quantum theory, but that, instead, the representation of the physical system by a quantum-mechanical state is a representation not of the actual world itself, but rather of the tencencies for the occurrence of an actual event that will select and actualize one of the macroscopically distinct alternatives.

In the context of the Libet experiments the critical point is that according to the Heisenberg picture there must first be a separation, generated by the evolution in accordance with the deterministic equation of motion, of the physical state into parts representing several macroscopically distinct possibilities before the act of choosing one of these macroscopically distinct alternatives occurs. In the brain most of the processing activity is done at the unconscious level: the lower-level process first prepares the distinctive alternatives, and the Heisenberg actual event then selects and actualizes one of them. Thus the delay found by Libet is demanded by this quantum mechanical theory of consciousness.

In the homuncular theory it would seem that the hornunculus could first decide to raise the arm, and then interact with the brain in order to bring about its desired end, and that the conscious event would therefore precede the neural activity that leads to the motor action.

\subsection{Comparison to Bohr}

The strictly orthodox Copenhagen interpretation of quantum theory brings human experience into physics in a way much more explicit than classical tileory did. The quantum theory is interpreted as fundamentally a theory that allows the scientist to form expectations about certain of his experiences. These are experiences that can be described in terms of specifications formulated in terms of the concepts of classical physics. This last stipulation effectively removes the in Lividual human experience from any place of prominence, for it makes the referents of the theory a class of external facts that all observers generally agree upon. So, in the end, the role of the subjective observer is no different than it was in classical physics: he is the subjective observer of essentially objective external facts. The issue of the connection of brain processes to mental process 
is thus never brought into question. In fact, this issue is moved by Bohr outside the domain to which quantum theory might apply by raising certain objections in principle to the application of quantum theory to biological systems.

In Bohr's words: "The incessant exchange of matter which is insefarably connected with life will even imply the impossibility of regarding an organism as a well-defined system of material particles like the system considered in any account of the ordinary and physical chemical properties of matter. In fact, we are led to conceive the proper biological regularities as representing laws of nature complementary to the account of properties of inanimate bodies ... ${ }^{n 29)}$.

The problem behind these words is that the interaction of a quantum system with its environment introduces conceptual difficulties that are, in fact, much more severe than those of classical physics. In classical physics when a particle leaves the system ind becomes part of the environment it leaves the system $i$. a state that is well defined in principle. In quantum theory this is not tho aase. The state of the residual system alone is not well defined: one must, in principle, for a compleie description, keep track of each particle that has lef; the state of the residual part depends on the location of each particle that has left, but each such location is defined only as a smeared-out superposition of possibilities. This means that each current brain state is not a single state in which the parts have well-defined locations, but is rather a superposition of states in which the parts have locations that depend on the ill-defined locations of the many particles that have long since left the brain and body. But what thought can be associated with surh a smeared- - ' superposition of brain states?

As Bohr emphasizes, some new ideas are needed: the strictly orthodox interpretation of quantum theory gives neither a practically useful nor conceptually cogent picture of what is going on in brains. The Heisenberg ontology, provides the simplest cogent extention of the strictly orthodox position. In it the actual brain events constitute a closely packed sequence of events that continually defined the key macroscopic features of the brain state.

\subsection{Comparison to von Neumann and Wigner}

von Neumann's analysis of the process of measurement involves a sequence of measuring devices, each of which detects the result of a measurement performed by the device prior to it in this sequence, with the final "device" lying 
deep within the brain. von Neumann accepted a principle of "pscho-physical parallelism ${ }^{n}$, which asserts that the process of subjective perception has a counterpart in the objective physical world, described in ordinzry space.

von Neumann's colleague, Eugene Wigner, elaborateu upon this idea, suggestin', rather, a reciprocal interaction between mind and matter. ${ }^{30)}$ However, in his later works ${ }^{31}$ ) Wigner rejected the idea that unmodified orthodox quantum theory can be applied to macroscopic systems. He, like Bohr, cited the important effects of interactions with an uncontrollable environment.

It is worth emphasizing that in the proposal being advanced here the actual events associated with human conscious experiences are not presumed to be the only actual events: actual events associated for example with the firing of a Geiger counter are presumed to exist, as Heisenberg assumed. Here it is merely accepted that, under similar conditions, the brain, which also is a physical system, should also be subject to the collapsing action of actual events.

\section{Related Philosophical Issues}

The success of classical physics in earlier centuries gave credence to the Newtonian idea of the universe as a machine, and to the concomitant Cartesian idea of consciousness as an impotent witness to a pre-ordained course of events. The rise of quantum theory in this century modified the Cartesian idea only slightly. In the absence of a quantu-n-mechanical treatment of the brain, consciousness became, instead, an impotent witness to a whimsical course of events. This constitutes no basic change in the Cartesian conception of the role of consciousness.

This Cartesian idea, backed by the authority of science, has exerted an enormous influence on philosophy, and a corrosive influence on the philosophical foundations of human values. On the other hand, the quantum theory of consciousness described above will, if validated by ongoing empirical studies, constitute a scientifically supported alternative to the Cartesian ontology. It will, as such, have far-reaching philosophical ramifications. Two of these are briefly mentioned.

\subsection{The Efficacy of Consciousness}

In Heisenberg's ontology the actual event is efficacious: it aciualizes one localized macroscopic pattern of activity from among a set of previously allowed 
possibilities. These possibilities, or, more preciseiy, the tendencies for the actualization of these alternative possible activities, are generated in a mathematically deterministic way by Heisenberg's equations of motion, which are the quantum analogs of corresponding classical equations of motion.

According to the theory advanced here each actual event has two aspects; a feel, and a physical representation within the quantum formalism. The feel is asserted to be a veridical image of the effect of the action of the physically described event.

At the purely physical level the Heisenberg actual event is passive: it is simply the coming into being of a new set of tendencies. However, in the context of the present ontology the actual event must be construed actively: the event actualizes the shift in teridencies. If the feel is identified as the active aspect of the event then the feel is the veridical feel of actively actualizing the new state of affairs, and consciousness becomes the efficacious agent that it veridically feels itself to be.

\subsection{The Quantum Choice}

The question arises: What determines which of the alternative possible brain activities is actualized by an actual event?

According to contemporary quantum theory, two factors contribute to this quantum choice. The first is the local deterministic evolution of tendencies governed by the Heisenberg equation of motion. This factor brings in all of the local historical influences such as heredity, learning, reflective contemplation on priorities and values, etc., that contribute to the formation of the current state of the brain. These factors determine, however, only the tendencies, or weights, associated with the various possible distinct courses of action. Then an actual event occurs. This event actualizes one of the distinct top-level patterns of brain activity, and bence selects one these distinct possible courses of action. This selection is, according to contemporary quantum theory, made by the second factor: pure chance.

Pure brute stochasticity, with no ontological substrate, is in my opinion an absurdity: the statistical regularities must bave some basis. On the other hand, the answer provided by contemporary quantum theory is probably correct in the sense that the basis for the quanturn choices cannot be conceptualized in 
terms of the ideas that it employs. Within that framework these choices must therefore appear to come out of nowhere; they must be, in the word used by Pauli and by Bohr, "irrational".

This inadequacy of the usual concepts can, I believe, be deduced by attending to certain features of the mathematical structure of the quantum formalism itself. The Heisenberg ontology is a kind of pictorial representation of this mathematical structure. It has, however, one exceedingly strange feature. This feature is superficially similar to the correlation effects that occur in classical statistical mechanics. Classically, if two systems become statistically correlated, due to some interaction between them, and each of them subsequently moves to one of two regions that are spatially well separated, then a measurement on one of the systems can provide statistical information about the other system, even though the two systems are far apart. There is nothing strange about this. However, if the statistical weights are interpreted as "objective tendencies", which have objective existence, which is the basic idea of Heisenberg's ontology, then the change in the far-away statistical properties as a consequence of a measurement performed here would constitute an instantaneous action-at-a-distance.

The Heisenberg ontology manifests precisely such an action-at-a-distance, and hence would seem to be unacceptable. At least it seemed to be unacceptable until the work of J.S. Bell in 1964..$^{32)}$ That work, suitably reformulated ${ }^{33)}$, shows, however, that if the choices between macroscopically distinct alternatives, such as the firing or nonfiring of a Geiger counter, are indeed made by nature, as the Heisenberg ontology maintains (in opposition to the many-worlds view, which maintains that both alternatives occur, but in noncommunicating branches of the universe) then these choices cannot be implemented by local actions: they can be implemented only by actions that transcend spacetime separation; i.e., that can act without attenuation over large space-like distances.

The conclusion, here, is that if the many-world idea is incorrect, and the macroscopic world is therefore roughly what it appears to be, then the structure of the predictions of quantum theory itself demands that the basic process of nature be intrinsically global: it cannot respect spatial separations in the way that familiar causal processes do. Thus to the extent that we confine our thinking to processes of the familiar local kind the quantum choice must appear to come from nowhere. 
The implication of the foregoing considerations is that although the flow of conscious events associated with a particular human brain bas important personal aspects, which arises from the fact that the content of these events is the feel of the acts of manipulation of the web of symbols created by the brain upon that web itself,nevertheless the fundamental process that is expressing itself through these local events is intrinsically global in character: it cannot be understood as being localized in the brain, or in the body. Rather it must act in a coordinated way over much of space. Neither contemporary science nor the present work addresses the issue of how that global process works. Our ignorance concerning this intrinsically global process is represented in these theories by the introduction of "pure choice".

\section{Summary}

The quantum theory of consciousness developed here :

1. Makes consciousness efficacious.

2. Rests directly on the mathematical formalism of quantum theory.

3. Parsimoniously accepts no kinds of entities not present in the Heisenberg or Copenhagen conceptions of nature.

4. Adheres fully to quantum thinking.

5. Meets the Einstein demand that basic physical theory describe the processes of nature, not merely our knowledge of those processes.

6. Mends the Cartesian cut by identifying an entity, the Heisenberg actual event, that unites as its two faces the subjective and objective aspects of mindbrain action.

7. Enunciates a principle of mind-brain isomorphism that seems able to account for the full content and structure of felt human experience, and its connection to brain process.

8. Identifies the "self" as a slowly evolving background component of human experience, not as the owner of that experience.

9. Describes the consciousness of man as a localized aspect of a global integrative process. 


\section{References}

1. William James, The Principles of Psychology, Dover, New York, 1950, Vol. I p. 146.

2. William James, Psychology: Briefer Course, Henry Holt, New York, 1983, p. 468.

3. Werner Heisenberg, Physics and Philosophy, Harper and Rowe, New York, 1958, Chapt. III.

4. Gerald Edelman, The Remembered Present: A Biological Theory of consciousness, Basic Books, New York, 1990; Neural Darwinism: The Theory of Neuronal Group Selection, Basic Books, New York, 1987.

5. Aaron Fogelson and Robert Zucker, Presynaptic Calcium Diffusion from Various Arrays of Single Channels: Implications for Transmitter Release and Synaptic Facilitation, Biophys. J, 48, 1003-1017, 1985.

6. Henri Korn and Donald Faber, Regulation and Significance of Probabilistic Release Mechanisms at Central Synapses, in Synaptic Function, eds. Edelman, Gall, and Gowan, Wiley, New York, 1987.

7. Lester Ingber, Statistical Mechanics of Neocortical Interactions Dynamics of Synaptic Modification. Phys. Rev. A8, 385-416, 1983.

8. John von Neumann, Mathematical Foundations of Quantum Mechanics, Princeton University Press, 1955, Chapt. VI.

9. William James, The Principles of Psychology, Vol. one, (Reprint of 1890 Text) Dover, New York, 1950, p. 241.

10. Ibid, p. 178

11. Ibid, p. 606

12. Ibid p. 612

13. Ref. 4, pp.93-94. 
14. ibid, p. 56, pp. 109-112.

15. ibid, p. $93,57$.

16. ibid, p. 93.

17. ibid, p. 97.

18. ibid, p. 155.

19. ibid, p. 101.

20. ibid, n. 100.

21. ibid, p. 10 .

22. ibid, p. 19, 253.

23. ibid, p. 19, 260.

24. ibid, p. 10.

25. John C. Eccles, Proc. R. Soc. Lond B227, 411-428 (1986); A Unitary hypothesis of mind-brain interaction in the cerebral cortex: dendrons, psychous, the 5th Dennis Gabor Lecture; The Microsite Hypothesis of the Mind-Brain Problem, in this volume.

26. B. Williams, Brain Damage, Behavior and the Mind, New York, Wiley, 1976; D.L. Schacter, M. P. Mc Andrews, and M. Moscovich, Access to consciousness: Dissociations between implicit and explicit knowledge in neurophysiological syndromes, in Thought Without Language, ed. L. Weiskrants 242-78, Oxford: Clarendon, 1988; Karl Pribram, Ref. 22 Ch.6.

27. Karl Pribram, Brain and Perception, 1990. Professor Pribram, commenting on my paper, says "there is good evidence that the brain process responsible for updating are coextensive with those involved in processing conscious awareness of the projected body-world schema. This reflective aspect of 'self-consciousness' is called intentionality by philosophers in [and] is dependent upon paying attention ...". My theory claims, essentially, 
that all conscious awareness is a generalization of this kind of consciousness. Professor Pribram also suggests that my term "symbol" be replaced by the term "action pattern", to distinguish it from the AI use of the term "symbol". He also affirms that there is a wealth of neurophysiological and neuropsychological date to support my picture of brain organization in terms of a top-level process that issues instructions to lower-level processes as described in the text.

28. Benjamin Libet, Cerebral "time-on" theory of conscious and unconscious mental function, in this volume.

29. N. Bohr, Atomic Physics and Human Knowledge, John Wiley, New York, 1959 p.20-21.

30. E. Wigner, in the Scientist Speculates, I.J. Good (Ed.) Windmill Press, Surrey England (1967).

31. E. Wigner, in Quantum Optics, Experimental Gravitation, and Measurement Theory, NATO AS1 Series, Series B: Physics, Vol. 94.

32. J.S. Bell, Physics 1, 1964.

33. H.P. Stapp, EPR and Bell's Theorem: A Critical Review. To be published in Foundations of Physics. 

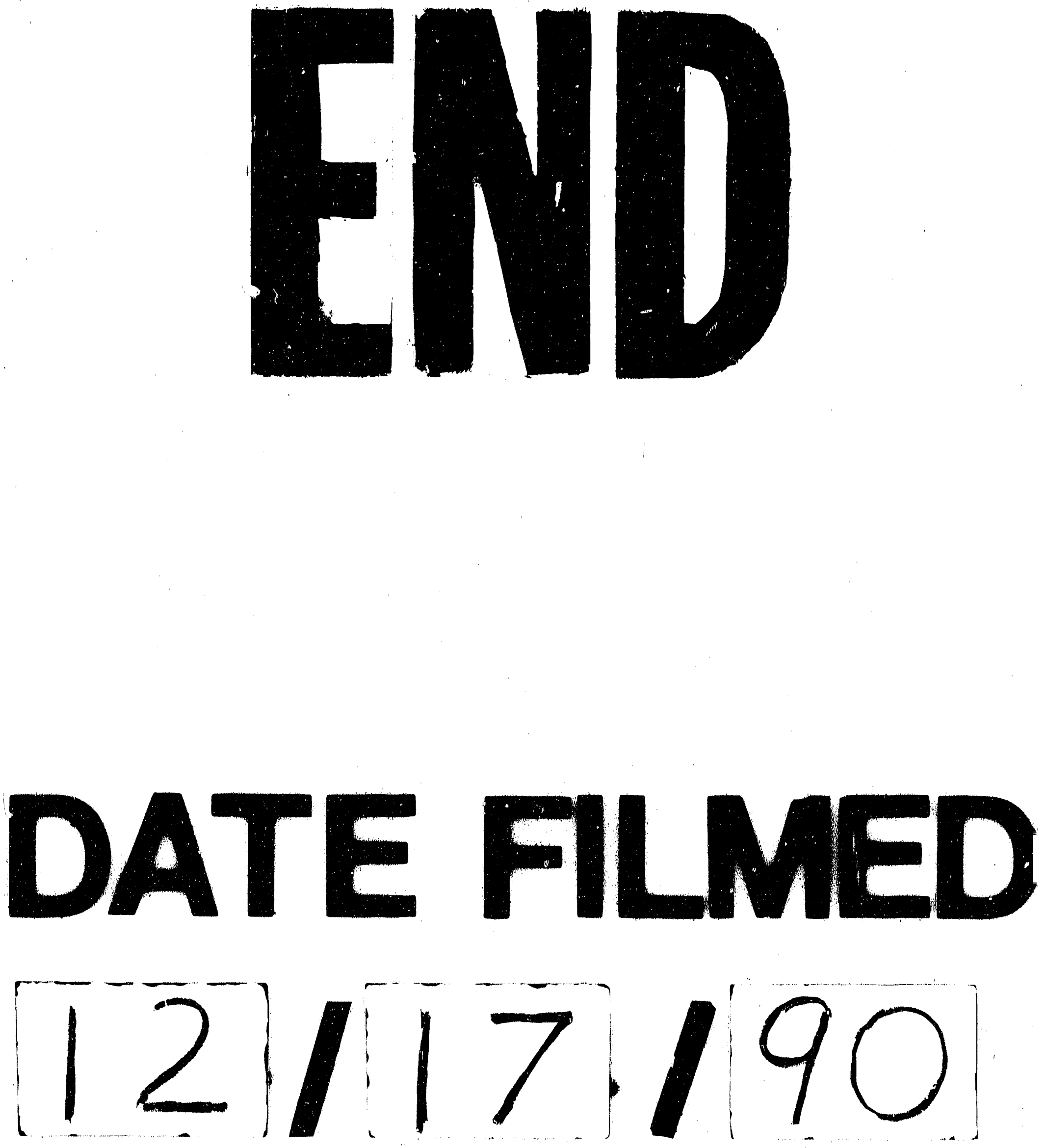
